Quim. Nova, Vol. 34, No. 4, 700-703, 2011

\title{
PRECONCENTRACIÓN Y ATOMIZACIÓN CON TUBO EN LA LLAMA PARA LA DETERMINACIÓN DE CADMIO EN AGUA DE MAR
}

\author{
Elizabeth González Sepúlveda* y Andrea Artigas Fuentes \\ Departamento de Química Ambiental, Facultad de Ciencias, Universidad Católica de la Santísima Concepción, Caupolicán 491, \\ Concepción, Chile \\ José Neira Hinojosa \\ Departamento de Análisis Instrumental, Facultad de Farmacia, Universidad de Concepción, Calle Edmundo Larenas 183, \\ Concepción, Chile
}

Recebido em 30/6/10; aceito em 5/10/10; publicado na web em 7/2/11

\begin{abstract}
ON-LINE PRECONCENTRATION SYSTEM COUPLED TO THERMOSPRAY FLAME FURNACE-AAS FOR CADMIUM

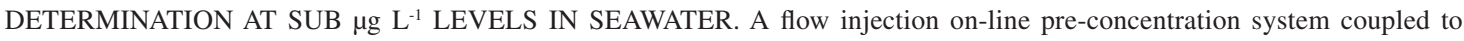
thermospray flame furnace atomic absorption spectrometry (TS-FF-AAS) for cadmium determination at sub $\mu \mathrm{L} \mathrm{L}^{-1}$ levels in seawater samples was developed. The on-line system was evaluated by analysing cadmium containing in a synthetic seawater matrix $(2.5 \% \mathrm{~m} / \mathrm{v}$ $\mathrm{NaCl}, 0.5 \% \mathrm{~m} / \mathrm{v} \mathrm{MgCl}$ and $0.8 \% \mathrm{~m} / \mathrm{v} \mathrm{CaCl}_{2}$ ). A sample volume of $2 \mathrm{~mL}$ allows determining Cd with a detection limits of $30 \mathrm{ng} \mathrm{L}^{-1}$ (3* $\sigma_{\text {blank }} /$ slope), pre-concentration factor of 34 and repeatability of $1,8 \%$ (calculated as RSD, N=8 and containing $200 \mathrm{ng} \mathrm{L}^{-1} \mathrm{of} \mathrm{Cd}$ ).
\end{abstract}

Keywords: cadmium; seawater; TS-FF-AAS.

\section{INTRODUCCION}

En los últimos años se han reportado mejoras significativas en los límites de detección de la espectrofotometría de la absorción atómica (AAS) mediante atomización con tubo en la llama en sus variantes denominadas thermospray flame furnace atomic absorption spectrometry (TS-FF-AAS) $)^{1-4}$ y beam-injection flame furnace atomic absorption spectrometry (BIFF-AAS).$^{5-7}$ Dentro de estas mejoras, Wu et al. ${ }^{4}$ ha propuesto una nueva forma de introducción de muestra en TS-FF-AAS y Lobo et al., ${ }^{8}$ ha presentado importantes resultados del uso de la mezcla de gases de óxido nitroso-acetileno para la determinación de estaño. Por otro lado, variadas aplicaciones de la TS-FF-AAS han sido investigadas, tales como: las determinaciones de los metales trazas $\mathrm{Cd}, \mathrm{Pb}$ y $\mathrm{Zn}$ tanto en muestras biológicas ${ }^{9,10}$ y alimentos, ${ }^{11-13}$ como también en algunas matrices marinas ${ }^{14,15}$ y Cd y $\mathrm{Pb}$ en muestras de tabaco brasilero. ${ }^{16}$

En agua de mar costera, marismas y aguas oceánicas, la contaminación por metales pesados, principalmente por $\mathrm{Cd}, \mathrm{Pb}$ y $\mathrm{Hg}$, genera gran preocupación por su alta toxicidad y se ha hecho necesario sus determinaciones con técnicas cada vez más sensibles y confiables. ${ }^{9}, 14$ De estos tres metales, el $\mathrm{Cd}$ es uno de los metales pesados que se encuentra en más baja concentración, aproximadamente entre 0,01 a $0,2 \mu \mathrm{g} \mathrm{L}^{-1}$, y su determinación directa por AAS con llama constituye un gran desafío debido a que la matriz de las sales disueltas (principalmente $\mathrm{NaCl}$ entre 2-3\%) interfiere significativamente en su análisis. Sobre esto último, en un trabajo anterior ${ }^{14}$ relacionado con el análisis directo de los metales $\mathrm{Cd}, \mathrm{Pb}$ y $\mathrm{Zn}$ en agua de mar, se encontró que por TS-FF-AAS sólo fue posible la determinación confiable de $\mathrm{Zn}$ a

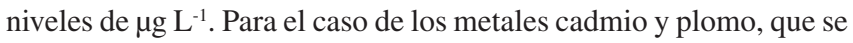
encuentran en concentraciones más baja, la alta interferencia de las sales de $\mathrm{NaCl}$ no permite la determinación directa de estos metales en dichas aguas costeras. Una alternativa para suplir este problema analítico ha sido el desarrollo y aplicación de metodologías analíticas más sensibles y confiables, por ejemplo la pre-concentración/ separación de la matriz, ${ }^{17,18}$ co-precipitación, ${ }^{19}$ extracción, ${ }^{20,21}$ etc., todo ello con el fin de separar la matriz interferente y mejorar significativamente los límites de detección en técnicas como absorción atómica con 1lama, ${ }^{22-24}$ horno de grafito, ${ }^{25-27}$ plasma óptico acoplado inductivamente ${ }^{24-26}$ y plasma masa acoplado inductivamente. ${ }^{28}$ Burguera et al. ${ }^{29}$ han presentado los avances en sistemas de inyección en flujo que incorpora la preconcentración y el empleo de variadas técnicas para la determinación de metales pesados a niveles de ng $\mathrm{L}^{-1}$ en materiales biológicos, aguas dulces y aguas de mar. En cuanto a estudios de preconcentración y separación de matriz acoplado a TS-FF-AAS, se ha reportado la performance en la determinación de $\mathrm{Cd}$ y $\mathrm{Pb},{ }^{30}$ la determinación de $\mathrm{Cu}$ en muestras de aguas, ${ }^{31}$ y la determinación de $\mathrm{Cd}$ en materiales biológicos certificados. ${ }^{32}$

En el campo de la oceanografía la preconcentración con la resina Chelex 100 ha sido aplicada por más de 35 años para la determinación de metales pesados en agua de mar. ${ }^{33,34}$ Es así que en análisis rutinario sobre un $70 \%$ de las determinaciones de metales pesados son aún realizados en sistemas batch y empleando dicha resina. ${ }^{35,36}$ Esta resina es de bajo costo y presenta como particularidad una buena eficiencia de preconcentración cuando es utilizada en grandes cantidades (sobre $2 \mathrm{~g}$ ) sin embargo, sus propiedades físicas y químicas son muy cambiantes por lo que normalmente suele usarse para un ciclo de preconcentración, siendo desechada posteriormente.

En este trabajo se presentan los arreglos experimentales de un sistema de preconcentración/separación con resina Chelex 100 en línea con la TS-FF-AAS para la determinación de Cd a niveles de sub $\mu \mathrm{g} \mathrm{L}^{-1}$ en muestras de aguas con alto contenido de $\mathrm{NaCl}$. Se informan los resultados de límite detección, límite de cuantificación, repetibilidad, factor de enriquecimiento y recuperación analítica de Cd en una muestra de agua de mar costera proveniente de la Bahía de Concepción-Chile. 


\section{PARTE EXPERIMENTAL}

\section{Reactivos}

Los reactivos utilizados fueron metanol, ácido nítrico concentrado, acetato de amonio y amoniaco, todos calidad suprapur de Merck. Se preparó soluciones patrones de $\mathrm{Cd}$ a partir de una solución titrisol Merck de $1000 \mu \mathrm{g} \mathrm{mL}^{-1}$. En la preparación de las soluciones estándares se utilizó agua desionizada calidad ultrapura de 18,2 M $\Omega$ cm (sistema Milli-Q, Millipore, Bedford M.A.). Como relleno para la preconcentración de Cd y separación de la matriz, se empleó la resina sódica Chelex 100 (50-100 mesh, forma sódica, laboratorios Bio-Rad, Sigma, USA).

\section{Preparación de soluciones estándares y muestra de agua de mar}

Las soluciones estándares de Cd fueron preparadas por dilución sucesiva a partir de soluciones patrón de Cd de $1000 \mu \mathrm{g} \mathrm{mL} \mathrm{mL}^{-1}$, y en una matriz de agua de mar sintética compuesta por $2,5 \% \mathrm{~m} / \mathrm{v} \mathrm{NaCl}$, $0,5 \% \mathrm{~m} / \mathrm{v} \mathrm{MgCl}_{2}$ y en $0,8 \% \mathrm{~m} / \mathrm{v} \mathrm{CaCl}_{2}$ (equivalente a $35 \%$ m/ $/ \mathrm{v}$ de solutos disueltos). Como blanco de muestra se utilizó esta misma matriz sintética de agua de mar.

Para la aplicación de la preconcentración implementada se consideró una muestra de agua de mar proveniente de la Bahía de Concepción-Chile, que fue obtenida mediante una botella Niskin (1,5 L) desde una estación de muestreo ubicada a $500 \mathrm{~m}$ de la costa y a 3 $\mathrm{m}$ de profundidad. Dicha muestra presentó un $\mathrm{pH}$ 7,9 y fue dividida en tres sub-muestras las cuales fueron almacenadas y congeladas en botellas de plásticos de alta densidad para su posterior análisis.

$\mathrm{Al}$ momento de realizar el análisis de $\mathrm{Cd}$ por preconcentración y TS-FF-AAS, las muestras se descongelaron y se irradiaron en un reactor de cuarzo con una lámpara ultravioleta de $\mathrm{Hg}$ de $150 \mathrm{~W}$ por un período de $40 \mathrm{~min}$.

\section{Instrumentación}

Se utilizó un espectrofotómetro de absorción atómica, GBS, modelo 902, equipado con corrector continuo de fondo con lámpara de deuterio y lámpara de cátodo hueco de Cd (228.8 nm), Photron PT y LTD y un quemador de premezclado (cabezal de $10 \mathrm{~cm}$, empleando una llama de acetileno (flujo de 3,0 $\mathrm{L} \mathrm{min}^{-1}$ ) y aire (flujo de $10 \mathrm{~L} \mathrm{~min}^{-1}$ ).

Para el proceso de atomización de la muestra se utilizó un tubo metálico (tubo Inconel diámetro interno: $10 \mathrm{~mm}$; largo: $13 \mathrm{~cm}$ ) con 6 orificios en la parte inferior y un orificio en el punto medio del tubo, donde se ubicó un tubo capilar cerámico, Omega (diámetro externo: $3,2 \mathrm{~mm}$; largo: $10 \mathrm{~cm}$ ), provisto de dos orificios internos de diámetro $0,5 \mathrm{~mm}$ cada uno, el cual se utilizó como medio para introducir la muestra con el analito de interés.

\section{Sistema de preconcentración en línea acoplado a TS-FF-AAS}

Para la preconcentración en línea (Figura 1) se utilizó una minicolumna compuesta por un tubo de teflón de largo $7 \mathrm{~mm}$ y de diámetro de $3 \mathrm{~mm}$, el cual fue llenado con $12 \mathrm{mg}$ de resina Chelex 100. Previo a su utilización, se hizo pasar con la ayuda de una bomba peristáltica y a un flujo de $0,5 \mathrm{~mL} \mathrm{~min}^{-1}$, las siguientes soluciones: $2 \mathrm{~mL}$ de agua desionizada, $2 \mathrm{~mL}$ de $\mathrm{HNO}_{3}$ de $0,69 \% \mathrm{v} / \mathrm{v}, 2 \mathrm{~mL}$ de agua desionizada conteniendo $0,5 \mathrm{~mL}$ de amoniaco $1 \mathrm{~mol} \mathrm{~L}^{-1}$, y finalmente $2 \mathrm{~mL}$ de agua desionizada. Para la determinación de $\mathrm{Cd}$ se realizaron previamente tres procesos, carga de la muestra, elución y regeneración. Para el proceso de elución se utilizó $\mathrm{HNO}_{3}$ como eluyente y en el proceso de regeneración de la minicolumna $5 \mathrm{~mL}$ de una solución buffer de acetato de amonio $1 \mathrm{~mol} \mathrm{~L}^{-1}+$ hidróxido de amonio $0,5 \mathrm{~mol} \mathrm{~L}^{-1}$. Tanto para la carga, elución, regeneración y transporte del analito se utilizó una bomba peristáltica de 6 rodillos (Ismatec, modelo MCP ISM726, Switzerland) a un flujo de $0,60 \mathrm{~mL} \mathrm{~min}^{-1}$.

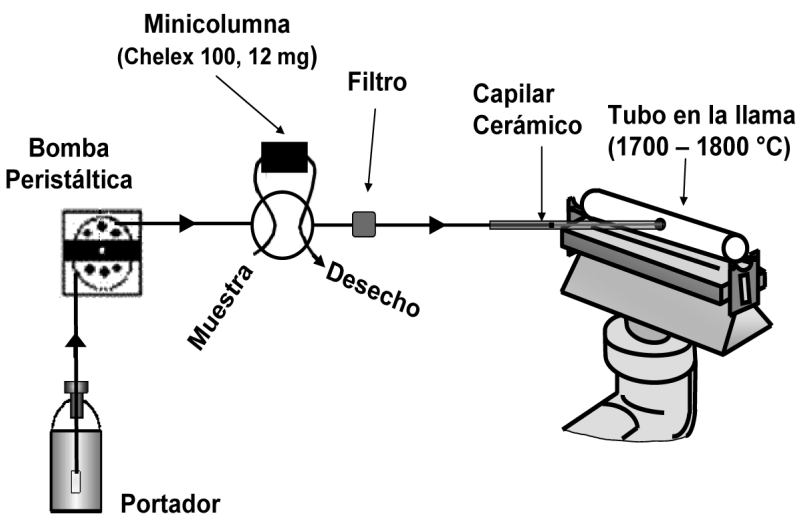

Figura 1. Diagrama esquemático del sistema de preconcentración/separación de matriz en la determinación de Cd en muestras de agua de mar costera

En el tratamiento de las señales analíticas el equipo de absorción atómica fue conectado a un computador a través de una interfase $\mathrm{e}^{37,38}$ y utilizado un software de adquisición y procesamiento de datos.

\section{RESULTADOS Y DISCUSION}

Se optimizó y evaluó para el sistema de preconcentración/separación las variables de vida útil de la resina, volumen de inyección de muestra, concentración de eluyente, y diámetro interno de la columna. Ensayos sucesivos de determinación de $\mathrm{Cd}$ conteniendo la matriz de agua de mar sintética, y en donde se usó como eluyente $\mathrm{HNO}_{3}(4,14 \% \mathrm{v} / \mathrm{v}), 12$ mg de resina Chelex 100, y usando un volumen de inyección de $2 \mathrm{~mL}$ demostraron que la resina pierde su eficiencia después de realizar 20 preconcentraciones. Considerando la menor anchura del pico de señal, se encontró que el volumen óptimo de inyección de muestra fue entre 2 a 3 $\mathrm{mL}$. Se realizó un estudio de influencia de la concentración del eluyente de $\mathrm{HNO}_{3}$. En base a los resultados obtenidos para los distintas concentraciones de eluyente, se propone el uso de una concentración del eluyente $\mathrm{HNO}_{3}$ de $0,69 \% \mathrm{v} / \mathrm{v}$, debido a que presenta una buena señal, efectiva para la remoción del analito retenido en la columna y que por el medio menos ácido proporciona una mayor vida útil de la resina de Chelex 100. Para evaluar las dimensiones óptimas de la microcolumna se consideraron dos columnas una de $9 \mathrm{~mm}$ de longitud y $2 \mathrm{~mm}$ de diámetro interno y otra de $7 \mathrm{~mm}$ de longitud y $3 \mathrm{~mm}$ de diámetro interno. Al comparar el funcionamiento de estas dos columnas en el sistema de preconcentración/ separación en línea para la determinación de Cd, la columna con un menor cociente $(\mathrm{L} / \mathrm{d})$, fue la que presentó mejores resultados. Debido a que se utilizó como relleno la resina Chelex 100, la cual tiene la característica de hincharse con facilidad y al estar en una columna de mayor longitud y de menor diámetro interno, la resina probablemente ejerce mayor presión en el flujo dificultando el paso de la muestra.

En la obtención de la típica curva de calibración por preconcentración/separación (Figura 2), se emplearon distintos estándares de Cd (200, 400, 600 y $\left.800 \mathrm{ng} \mathrm{L}^{-1}\right)$ todos contenidos en la matriz sintética de agua de mar. El sistema preconcentración permite obtener un límite de detección de $30 \mathrm{ng} \mathrm{L}^{-1}$ y de cuantificación de $115 \mathrm{ng} \mathrm{L}^{-1}$, una muy buena repetibilidad (1,8\% expresado como coeficiente de variación, para $\mathrm{Cd}$ de $200 \mathrm{ng} \mathrm{L}^{-1}$ y $\mathrm{N}=8$ ) y una vida útil de la columna cercana a 20 inyecciones sucesivas.

Basado en el cálculo de las pendientes de las curva de calibración con y sin preconcentración, se obtuvo un factor de enriquecimiento (FE) igual a 34 y un índice de consumo (CI) de 0,058. Aunque el FE 


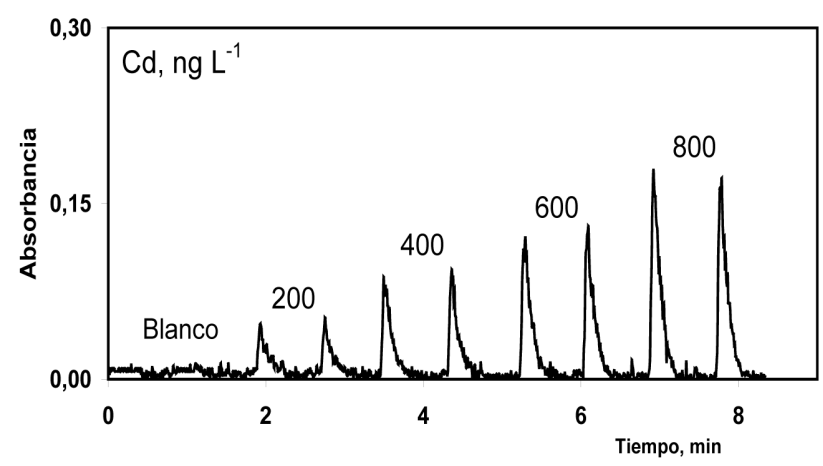

Figura 2. Típica curva de calibración obtenida para Cd por preconcentración/separación. Portador: $\mathrm{HNO}_{3}$ 0,69\% v/v + 5\% v/v metanol; volumen de inyección de muestra: 2,0 $\mathrm{mL}$

es indispensable para la evaluación de sistemas de preconcentración, este factor no proporciona información sobre la eficiencia del sistema, debido a que los altos valores de FE pueden ser alcanzados usando grandes tiempos de preconcentración y consumir de esta forma un gran volumen de muestra. ${ }^{36}$ Debido a esto se sugiere el uso de la eficiencia de concentración para la evaluación de este sistema. La eficiencia de concentración de Cd para este sistema de preconcentración/separación resultó igual a $1190 \mathrm{~h}^{-1}$. En relación a lo anterior, si al relacionar la misma cantidad y tipo de relleno de Chelex 100, y el mismo volumen de inyección para la preconcentración de $\mathrm{Cd}$, la eficiencia de concentración y el factor de enriquecimiento son concordantes con los obtenidos por Fang et al ${ }^{39}$ (valor eficiencia de $1200 \mathrm{~h}^{-1}$ y factor de enriquecimiento de 30). Esta buena comparación de la eficiencia de concentración y factor de enriquecimiento, acredita el buen desarrollo y aplicación del sistema de preconcentración con Chelex 100 para la determinación de Cd por TS-FF-AAS. Respecto a lo mencionado anteriormente, la mayoría de las investigaciones de determinación de cadmio por preconcentración y empleo de la TS-F-FAAS están basados en muestras que no reviste mayor problema por efecto de la matriz, y también por la digestión a que son sometidas, por ejemplo en tabaco, ${ }^{16,32}$ arroz, ${ }^{40}$ orina, ${ }^{41}$ agua mineral, ${ }^{32,42}$ repollo, ${ }^{10}$ agua de grifo y de ríos, ${ }^{32}$ etc.. En la presente investigación se realizó la determinación directa de $\mathrm{Cd}$ en muestras de agua de mar con un alto contenido salino (principalmente dado por $\mathrm{NaCl}$ ), que bajo éstas condiciones presenta una buena eficiencia en la preconcentración de Cd junto con la separación de la matriz, con límites de detección y repetibilidad comparables al obtenido en muestra con baja interferencia de la matriz (i. e. tabaco, arroz, orina, agua mineral, repollo, agua de grifo y ríos, etc).

La preconcentración en línea, descrito anteriormente, permite la determinación de $\mathrm{Cd}$ a niveles sub-ultra-trazas $\left(\mathrm{ng} \mathrm{L}^{-1}\right)$ en muestras de agua de mar, utilizando la resina quelante, Chelex 100. Así, se determinó por adición estándar múltiple para una muestra de agua de mar (Figura 3) proveniente de la Bahía Concepción-Chile la concentración de $\mathrm{Cd}$ fue de $153 \pm 8 \mathrm{ng} \mathrm{L}^{-1}$ (promedio de tres determinaciones de muestras independientes), valor que se encuentra en el rango reportado por Carrera et al. ${ }^{43}$ Por otro lado, basado en esta misma muestra de agua de mar y considerando siempre una recarga de $100 \mathrm{ng} \mathrm{L}^{-1}$ de $\mathrm{Cd}$ permitió obtener un porcentaje de recuperación promedio de $93 \pm 1 \%(\mathrm{~N}=5)$. Este porcentaje de recuperación es comparable con lo obtenido por Quiang et $a l .{ }^{44}$ y Vereda et $a l .{ }^{45}$ los cuales reportan respectivamente valores de porcentaje de recuperación de 96,6 y 94\%.

\section{CONCLUSIONES}

El desarrollo del sistema de preconcentración/separación de la matriz en línea con la espectrofotometría de absorción atómica con

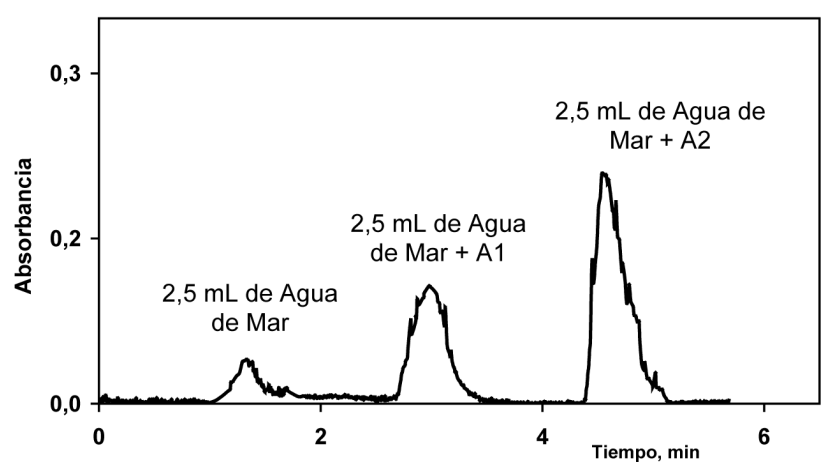

Figura 3. Adición estándar para la determinación de Cd en agua de mar costera. A1: adición de Cd $400 \mathrm{ng} \mathrm{L}^{-1}$ : A2: adición de Cd de $800 \mathrm{ng} \mathrm{L}^{-1}$. Portador: $\mathrm{HNO}_{3}$ $0,69 \% v / v+5 \% v / v$ metanol; volumen de inyección de muestra: $2,5 \mathrm{~mL}$

tubo en la llama permite la determinación de $\mathrm{Cd}$ a niveles de subultratrazas en muestras con un alto contenido de $\mathrm{NaCl}(2,5 \% \mathrm{~m} / \mathrm{v}$ $\mathrm{NaCl})$. El sistema se aplicó exitosamente en la determinación de $\mathrm{Cd}$ en una muestra de agua de mar costera proveniente de la bahía de Concepción-Chile.

\section{AGRADECIMIENTOS}

Los autores agradecen el apoyo de la Facultad de Ciencias y de la Dirección de Investigación de la Universidad Católica de la Santísima Concepción. Especial agradecimiento a la Dirección de Investigación de la Universidad de Concepción.

\section{REFERENCIAS}

1. Gáspar, A.; Széles, É.; Berndt, H.; Anal. Bional. Chem. 2002, 372, 136.

2. Davies, J.; Berndt, H.; Anal. Chim. Acta 2003, 479, 215.

3. Jiang, Y.; Li, Y.; Yan, X. P.; Electrophoresis 2005, 26, 661 .

4. Wu, P.; Liu, R.; Berndt, H.; Lv, Y.; Hou, X.; J. Anal. At. Spectrom. 2008, 23,37 .

5. Neira, J.; Berndt, H.; Fresenius Z. Anal. Chem. 2000, 368, 649.

6. Ratka, A.; Berndt, H.; Anal. Bioanal. Chem. 2004, 378, 416.

7. Aleixo, P. C.; Santos, Jr., D.; Tomazelli, A. C.; Rufini, I. A.; Berndt, H.; Krug, F. J.; Anal. Chim. Acta 2004, 512, 329.

8. Lobo, F. A.; Villafranca, A. C.; Morales, M.; Eclética Química 2005, 30, 61.

9. Pereira-Filho, E. R.; Berndt, H.; Arruda, M. A. Z.; J. Anal. At. Spectrom. 2002, 17, 1308

10. Carqueija, F.; Almeida, M.; Microchim. Acta 2007, 159, 183.

11. Nascentes, C. C.; Arruda, M. A. Z.; Nogueira, A. R. A.; Nóbrega, J. A.; Talanta 2004, 64, 912.

12. Rossini, F.; Nascente, C. C.; Neira, J. Y.; Nóbrega, J. A.; Talanta 2007, 73,845 .

13. Schiavo, D.; Neira, J. Y.; Nóbrega, J. A.; Talanta 2008, 76, 1113.

14. González, E.; Ahumada, R.; Medina, V.; Neira, J.; González, U.; Quim. Nova 2004, 27, 873.

15. Ahumada, R.; González, E.; Neira, J.; Revista Investigaciones Marinas 2004, 32,1

16. Andrade, F. P.; Nascentes, C. C.; Costa, L. M.; J. Braz. Chem. Soc. 2009, 20, 1460

17. Boyle, E.; Edmond, J.; Adv. Chem. Ser. 1975, 147, 44

18. Jarvis, K. E.; Williams, J. G.; Alcantara, E.; Wills, J. D.; J. Anal. At. Spectrom. 1996, 11, 917

19. Vassileva, E.; Proinova, I.; Hadjiivanov, K.; Analyst 1996, 121, 607.

20. Amorim, F. A. C.; Ferreira, S. L. C.; Talanta 2005, 65, 960.

21. Tuzen, M.; Parlar, K; Soylak, M.; J. Hazard. Mater. 2005, 121, 79. 
22. Santos, E. J.; Herrmann, A. B.; Ribeiro, A. S.; Curtius, A. J.; Talanta 2005, 65, 593.

23. Peña, Y.; Paredes, B.; Ronodòn, W.; Burguera, J.; Rondòn, L.; Carrero, P.; Capote, T.; Talanta 2004, 64, 1351.

24. Santos, W. N. L.; Santos, C. M.; Ferreira, S. L. C.; J. Braz. Chem. Soc. 2005, 16, 727.

25. Wang, J. H.; Hansen, E. H.; Anal. Chim. Acta 2005, 24, 1.

26. Burguera, J.; Burguera, M.; Spectrochim. Acta 2001, 56, 1801.

27. Colbert, D.; Johnson, K. S.; Coale, K. H.; Anal. Chim. Acta 1998, 377, 255.

28. Wang, J. H.; Hansen, E. H.; Trends Anal. Chem. 2003, 22, 225.

29. Burguera, J.; Burguera, M. En Advances in Flow Analysis; Trojanowicz, M., ed.; Wiley-VCH Verlag GmbH \& Co. KGaA: Weinheim, 1998.

30. Ivanova, E.; Bendt, H.; Pulvermacher, E.; J. Anal. At. Spectrom. 2004, $19,1507$.

31. Teixeira, C.; Da Costa, E.; Domínguez, G.; Anal. Sci. 2005, 21, 1337.

32. Teixeira, C.; Barbosa, A.; Gava, M.; Costa, E.; Orival, P.; J. Anal. At. Spectrom. 2006, 21, 1305.

33. Riley, J. P.; Taylor, D.; Anal. Chim. Acta 1968, 40, 479.
34. Kiptoo, J. K.; Ngila, J. C.; Silavwe, N. D.; J. Hazard. Mater. 2009, 172, 1163.

35. Pai, S.; Whung, P.; Lai, R.; Anal. Chim. Acta 1988, 211, 257.

36. Pai, S. C.; Fang, T.; Marine Chemistry 1990, 29, 295.

37. Neira, J. Y.; Nobrega, J. A.; Labor. Rob. Autom. 1999, 11, 260.

38. Neira, J. Y.; Reyes, N.; Nóbrega, J. A.; Labor. Rob. Autom. 2000, 12, 246.

39. Fang, Z.; Ruzicka, J.; Hansen, E. H.; Anal. Chim. Acta 1984, 164, 23.

40. Wen, X.; Wu, P.; Xu, K.; Wang, J.; Hou, X.; Microchem. J. 2009, 91, 193.

41. Wu, P.; Zhang, Y.; Lv, Y.; Hou, X.; Spectrochim. Acta, Part B 2006, 61, 1310 .

42. Melo, L.; Almeida, M.; Arruda, M. A. Z.; Bruns, E. R.; Costa, S. L.; Sep. Sci. Technol. 2008, 43, 815.

43. Carrera, M.; Rodríguez, V.; Ahumada, R.; Valenta, P.; Rev. Biol. Mar. 1993, 28, 151.

44. Quiang, F.; Limin, Y.; Qiuquan, W.; Spectrosc. Lett. 2007, 40, 547.

45. Vereda, E.; Palomo, L.; Siles, M. T.; García, A.; Cano, J. M.; J. Anal. At. Spectrom. 2001, 16, 293. 